\title{
Two-loop results on the renormalization of vacuum expectation values and infrared divergences in the FDH scheme
}

\section{Christoph Gnendiger}

Institut für Kern- und Teilchenphysik, TU Dresden, Germany

\section{Adrian Signer}

Paul Scherrer Institut, CH-5232 Villigen PSI, Switzerland and

Physik-Institut, Universität Zürich, Switzerland

\section{Marcus Sperling}

Institut für Theoretische Physik, Universität Hannover, Germany

\section{Dominik Stöckinger*}

Institut für Kern- und Teilchenphysik, TU Dresden, Germany

E-mail: Dominik. Stoeckinger@tu-dresden.de

\section{Alexander Voigt}

Institut für Kern- und Teilchenphysik, TU Dresden, Germany

\begin{abstract}
Recent progress in the understanding of vacuum expectation values and of infrared divergences in different regularization schemes is reviewed. Vacuum expectation values are gauge and renormalization-scheme dependent quantities. Using a method based on Slavnov-Taylor identities, the renormalization properties could be better understood. The practical outcome is the computation of the $\beta$ functions for vacuum expectation values in general gauge theories.

The infrared structure of gauge theory amplitudes depends on the regularization scheme. The well-known prediction of the infrared structure in CDR can be generalized to the FDH and DRED schemes and is in agreement with explicit computations of the quark and gluon form factors. We discuss particularly the correct renormalization procedure and the distinction between $\overline{\mathrm{MS}}$ and $\overline{\mathrm{DR}}$ renormalization. An important practical outcome are transition rules between CDR and FDH amplitudes.
\end{abstract}

Loops and Legs in Quantum Field Theory - LL 2014,

27 April - 2 May 2014

Weimar, Germany

\footnotetext{
${ }^{*}$ Speaker.
} 


\section{Introduction}

Precision calculations in QCD, the electroweak Standard Model, or supersymmetric models require regularization and renormalization in intermediate steps. Here we discuss two recent works where progress in the understanding of fundamental issues and results of practical relevance were obtained. The topic of [1] is the renormalization properties of vacuum expecation values (vevs) in spontaneously broken gauge theories. A method was developed which leads to a better understanding of the divergent renormalization of vevs and their gauge and renormalization-scale dependence. As a practical result the two-loop renormalization group $\beta$-functions of vevs in general and supersymmetric gauge theories were obtained and made available for use in spectrum generators and explicit calculations.

In [2] the structure of infrared divergences was investigated in different regularization schemes. The recent progress on computations using unitarity-inspired methods or four-dimensional approaches highlights the importance of studying QCD amplitudes in regularization schemes which differ from conventional dimensional regularization (CDR). Ref. [2] studies the relation between CDR and the four-dimensional helicity (FDH) scheme. The central results are the proof that the infrared structure can be predicted in both schemes in a similar way, and that there are simple translation rules which convert an FDH-regularized amplitude into a CDR-regularized one. These results are similar to results of Ref. [3], and they can be viewed as a continuation of Refs. [4], where a systematic one-loop comparison of the CDR, HV, FDH, and DRED regularization schemes was carried out and an earlier factorization problem of DRED was resolved.

\section{Vacuum expectation values and their renormalization}

The renormalization of a scalar field $\phi$ and associated vev $v$ can generically be written as

$$
\phi+v \rightarrow \sqrt{Z} \phi+\sqrt{Z} \sqrt{\hat{Z}} v
$$

Here $Z$ is the usual field renormalization constant, and $\hat{Z}$ is an additional renormalization constant, which characterizes to what extent $v$ renormalizes differently from $\phi$. The field renormalization leads to an anomalous dimension $\gamma$ of the scalar field, and one can define a similar quantity $\hat{\gamma}$, which is defined via $\hat{Z}$ in the same way as $\gamma$ is defined via $Z$. The renormalization group $\beta$ function for the running vev in the $\overline{\mathrm{MS}}$ or $\overline{\mathrm{DR}}$ scheme is then given as

$$
\beta_{v}=(\gamma+\hat{\gamma}) v
$$

A surprising observation was made in the literature in Refs. [5]. In the minimal supersymmetric standard model (MSSM), there are two Higgs doublets $H_{u}, H_{d}$, and there is a cancellation between the two additional renormalization constants, i.e. $\delta \hat{Z}_{H_{u}}-\delta \hat{Z}_{H_{d}}=$ finite at the one-loop level. The usual field renormalization constants $\delta Z_{H_{u}}, \delta Z_{H_{d}}$ don't share this property. The implication is that the $\beta$ function for the parameter $\tan \beta=v_{u} / v_{d}$ can simply be written in terms of the usual anomalous dimensions, i.e.

$$
\delta \hat{Z}_{H_{u}}-\delta \hat{Z}_{H_{d}}=\text { finite } \quad \Rightarrow \quad \beta_{\tan \beta}=\left(\gamma_{H_{u}}-\gamma_{H_{d}}\right) \tan \beta
$$


at the one-loop level, with no $\hat{\gamma}$ contributions.

Furthermore, one may ask why the additional renormalization constant $\hat{Z}$ is necessary in the first place. Clearly, in non-gauge theories, i.e. theories with only a global symmetry, it is not required. The point is that local gauge theories can only be quantized using some kind of gauge fixing. Depending on which gauge fixing is chosen, the additional renormalization constant $\hat{Z}$ can be necessary. The method employed in Refs. [1] aims to make this explicit. In the process it explains the observation mentioned above, and it allows the explicit computation of $\beta_{v}$ at the two-loop level in generic gauge theories.

The essence of the method, introduced in Refs. [6] for a slightly different purpose, is to replace the vev by a background (classical) field $\hat{\phi}$, carry out the renormalization process (in particular the study of Slavnov-Taylor identities and their implications on the required independent renormalization constants) in presence of these background fields, and specialize to $\hat{\phi}=v=$ const only at the end. Using the background fields, the usual $R_{\xi}$ gauge fixing term for the abelian Higgs model can be written as

$$
F=\partial^{\mu} A_{\mu}+i e \xi\left(\hat{\phi}^{\dagger} \phi-\phi^{\dagger} \hat{\phi}\right)
$$

As long as $\hat{\phi}$ is treated as a background field which transforms covariantly under global gauge transformations, this gauge fixing term breaks only local, but not global gauge invariance. As a consequence only renormalization constants in agreement with global gauge invariance can be necessary. $\hat{Z}$ can appear as the field renormalization of $\hat{\phi}$. The main trick is to define a BRS transformation of the background field, as

$$
s \hat{\phi}=\hat{q}, \quad s \hat{q}=0 .
$$

Then the Slavnov-Taylor identity provides a useful relation for $\hat{Z}$ : this renormalization constant can be directly determined from the Green function $\Gamma_{\hat{q} K_{\phi}}$, because the counterterm contribution to this Green function is $\Gamma_{\hat{q} K_{\phi}}^{\mathrm{ct}}=-\frac{1}{2} \delta \hat{Z}$. Here $K_{\phi}$ is the source for the BRS transformation of $\phi$, so this Green function corresponds to the coupling of the composite operator $(s \phi)$ to the background field $\hat{q}$. It is an unphysical Green function but a very useful technical tool.

Fig. 1 (left) shows the single one-loop diagram in a generic gauge theory. The only vertex of $K_{\phi}$ follows from the structure of the BRS transformation of scalar fields: $K_{\phi}$ couples to one scalar field and one Faddeev-Popov ghost; the coupling is the gauge coupling. The only vertex of $\hat{q}$ originates in the gauge fixing: $\hat{q}$ couples to the prefactor of $\hat{\phi}$ in the gauge fixing, i.e. the Feynman rule is proportional to $\xi$ and to the gauge coupling. Hence the one-loop contribution to this Green function (and thus to $\delta \hat{Z}$ ) is proportional only to the gauge parameter $\xi$ and to the squared gauge coupling of the scalar field. In a generic gauge theory the $\overline{\mathrm{MS}}$ result can be written as

$$
\delta_{a b} \delta \hat{Z}^{(1)}(a)=\frac{1}{(4 \pi)^{2}} 2 g^{2} \xi C_{a b}^{2}(S) \cdot \frac{1}{\varepsilon},
$$

where $C_{a b}^{2}(S)=T_{a c}^{A} T_{c b}^{A}$ with the generators $T^{A}$ acting on the scalar fields $\phi_{a}$. Hence the result is proportional to $\xi$ and to the squared gauge couplings of the scalar field. Since all two-loop diagrams must involve the same two vertices as the ones in Fig. 1 (left), this proportionality extends to the two-loop result; Fig. 1 (right) shows a sample two-loop diagram. 

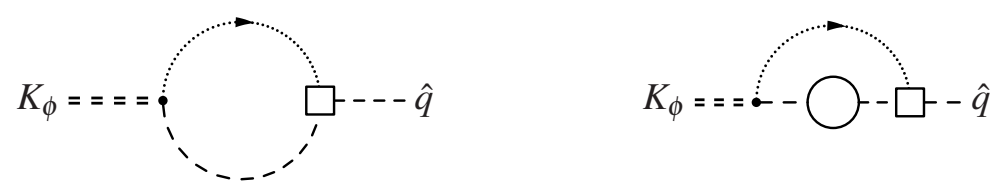

Figure 1: Left: The single one-loop diagram to $\Gamma_{\hat{q} K_{\phi}}$. The dotted line is a Faddeev-Popov ghost propagator; the dashed line a scalar field propagator. Right: Sample two-loop diagram with a fermion loop (solid line), which leads to corrections involving Yukawa couplings.

This result already explains two statements made earlier. First we see that in Landau gauge, where $\xi=0$, we obtain $\delta \hat{Z}=0$. This reflects the fact that in a gauge which does not break global gauge invariance, the additional renormalization constant $\hat{Z}$ is not needed. Second, for $\xi \neq 0, \hat{Z}$ is needed but is proportional to squared gauge couplings. This explains the observation (2.3) since the squared SU(2) and U(1) gauge couplings of the two Higgs doublets in the MSSM are equal.

The full two-loop result for $\delta \hat{z}, \hat{\gamma}$ and $\beta_{v}$ has been obtained in Refs. [1]. Here we provide the result for $\gamma$ and $\hat{\gamma}$ for general supersymmetric gauge theories:

$$
\begin{aligned}
\left.\gamma_{a b}^{(1)}(\mathrm{S})\right|_{\mathrm{SUSY}} ^{\overline{\mathrm{DR}}}= & \frac{1}{(4 \pi)^{2}}\left[g^{2}(1-\xi) C_{a b}^{2}(\mathrm{~S})-\frac{1}{2} Y_{a p q}^{*} Y_{b p q}\right], \\
\left.\hat{\gamma}_{a b}^{(1)}(\mathrm{S})\right|_{\mathrm{SUSY}} ^{\overline{\mathrm{DR}}}= & \frac{1}{(4 \pi)^{2}} 2 g^{2} \xi \xi^{\prime} C_{a b}^{2}(\mathrm{~S}), \\
\left.\gamma_{a b}^{(2)}(\mathrm{S})\right|_{\mathrm{SUSY}} ^{\overline{\mathrm{DR}}}= & \frac{1}{(4 \pi)^{4}}\left\{g^{4}\left[\left(\frac{9}{4}-\frac{5}{3} \xi-\frac{1}{4} \xi^{2}\right) C_{2}(\mathrm{G})-S_{2}(\mathrm{~S})\right] C_{a b}^{2}(\mathrm{~S})\right. \\
& -2 g^{4} C_{a c}^{2}(\mathrm{~S}) C_{c b}^{2}(\mathrm{~S})+\frac{1}{2} Y_{a r c}^{*} Y_{r p q} Y_{p q d}^{*} Y_{b c d} \\
& \left.+g^{2}\left[C_{a c}^{2}(\mathrm{~S}) Y_{c p q}^{*} Y_{b p q}-2 Y_{a p q}^{*} C_{p r}^{2}(\mathrm{~S}) Y_{b r q}\right]\right\}, \\
\left.\hat{\gamma}_{a b}^{(2)}(\mathrm{S})\right|_{\mathrm{SUSY}} ^{\overline{\mathrm{DR}} / \overline{\mathrm{MS}}}= & \frac{\xi \xi^{\prime}}{(4 \pi)^{4}}\left\{g^{4}\left[\frac{7-\xi}{2} C_{2}(\mathrm{G}) C_{a b}^{2}(\mathrm{~S})-2(1-\xi) C_{a c}^{2}(\mathrm{~S}) C_{c b}^{2}(\mathrm{~S})\right]\right. \\
& \left.-g^{2} C_{a c}^{2}(\mathrm{~S}) Y_{c p q}^{*} Y_{b p q}\right\} .
\end{aligned}
$$

Here $Y_{a b c}$ are conventionally normalized superpotential couplings. To our knowledge, the scalar field anomalous dimension $\gamma^{(2)}$ has not been provided in the literature before. We remark that this anomalous dimension is the one of the component scalar field in Wess-Zumino gauge, and it is not equal to the superfield anomalous dimension in a supersymmetric gauge fixing. From these results eq. (2.2) can be used to obtain the $\beta$ function for vevs and for related quantities such as $\tan \beta$. Refs. [1] provide explicit results for models of practical and phenomenological interest, such as the MSSM, the NMSSM, and the $\mathrm{E}_{6} \mathrm{SSM}$. The general results have been implemented in general programs such as Sarah [7] and FlexibleSUSY [8].

\section{Infrared structure of QCD amplitudes in the FDH scheme}

In recent years, new methods have been developed to compute gauge theory amplitudes, and the understanding of the infrared structure of these amplitudes has significantly improved. In particular, Refs. [9] have given a prediction for the infrared $1 / \varepsilon$ poles in conventional dimensional 
reduction for arbitrary QCD amplitudes. Specialized to form factors, this prediction reads

$$
\ln \mathbf{Z}=\left(\frac{\alpha_{s}}{4 \pi}\right)\left(\frac{\Gamma_{1}^{\prime}}{4 \varepsilon^{2}}+\frac{\Gamma_{1}}{2 \varepsilon}\right)+\left(\frac{\alpha_{s}}{4 \pi}\right)^{2}\left(-\frac{3 \beta_{20} \Gamma_{1}^{\prime}}{16 \varepsilon^{3}}+\frac{\Gamma_{2}^{\prime}-4 \beta_{20} \Gamma_{1}}{16 \varepsilon^{2}}+\frac{\Gamma_{2}}{4 \varepsilon}\right)+\ldots
$$

Here $\Gamma_{m}^{\prime}=-2 \gamma_{m}^{\text {cusp }} C_{q / g}, \Gamma_{m}=+2 \gamma_{m}^{i}$. with the cusp anomalous dimension $\gamma^{\text {cusp }}$ and parton anomalous dimension $\gamma^{i}$; the index $i$ refers to either quark or gluon, and the index $m$ refers to the coefficient of the respective quantity of $\left(\alpha_{s} / 4 \pi\right)^{m} ; \beta_{20}$ is the $\left(\alpha_{s} / 4 \pi\right)^{2}$-coefficient of the $\beta$ function for $\alpha_{s}$.

In view of the new computational methods based on unitarity, helicity and 4-dimensional algebra it is of high interest to study how the infrared structure depends on the regularization scheme, in particular how it is modified in schemes such as the four-dimensional helicity scheme (FDH) and dimensional reduction (DRED). An independent reason to study these regularization schemes is supersymmetry, which is broken in CDR, while FDH and DRED preserve supersymmetry to a large extent. In Refs. [4] the differences between these schemes were clarified and it was shown that all these schemes are consistent regularization schemes. In particular one-loop results of the earlier literature were shown to be consistent among each other and consistent with infrared factorization, if the schemes are used appropriately. The necessity to treat renormalization in the FDH scheme in the way advocated in [4] was also reiterated in Ref. [11].

Ref. [2] can be viewed as a continuation of Refs. [4] to the two-loop level and as an extension of Eq. (3.1) to other regularization schemes. Extending the infrared prediction (3.1) to FDH and DRED is possible by taking into account the main insight of Ref. [4], which is that FDH and DRED should be viewed as dimensional regularization with a new type of parton, a scalar field with multiplicity $N_{\varepsilon}=2 \varepsilon$, the $\varepsilon$-scalars. The $\varepsilon$-scalars have couplings and anomalous dimensions which differ from the ones of the gluons. At the two-loop level the only new coupling appearing in the infrared prediction is $\alpha_{e}$, the coupling of $\varepsilon$-scalars to quarks. The FDH result corresponding to Eq. (3.1) is

$$
\begin{aligned}
\ln \overline{\mathbf{Z}}= & \left(\frac{\alpha_{s}}{4 \pi}\right)\left(\frac{\bar{\Gamma}_{10}^{\prime}}{4 \varepsilon^{2}}+\frac{\bar{\Gamma}_{10}}{2 \varepsilon}\right)+\left(\frac{\alpha_{e}}{4 \pi}\right)\left(\frac{\bar{\Gamma}_{01}^{\prime}}{4 \varepsilon^{2}}+\frac{\bar{\Gamma}_{01}}{2 \varepsilon}\right) \\
& +\left(\frac{\alpha_{s}}{4 \pi}\right)^{2}\left(-\frac{3 \bar{\beta}_{20} \bar{\Gamma}_{10}^{\prime}}{16 \varepsilon^{3}}+\frac{\bar{\Gamma}_{20}^{\prime}-4 \bar{\beta}_{20} \bar{\Gamma}_{10}}{16 \varepsilon^{2}}+\frac{\bar{\Gamma}_{20}}{4 \varepsilon}\right) \\
& +\left(\frac{\alpha_{s}}{4 \pi}\right)\left(\frac{\alpha_{e}}{4 \pi}\right)\left(-\frac{3 \bar{\beta}_{11}^{e} \bar{\Gamma}_{01}^{\prime}}{16 \varepsilon^{3}}+\frac{\bar{\Gamma}_{11}^{\prime}-4 \bar{\beta}_{11}^{e} \bar{\Gamma}_{01}}{16 \varepsilon^{2}}+\frac{\bar{\Gamma}_{11}}{4 \varepsilon}\right) \\
& +\left(\frac{\alpha_{e}}{4 \pi}\right)^{2}\left(-\frac{3 \bar{\beta}_{02}^{e} \bar{\Gamma}_{01}^{\prime}}{16 \varepsilon^{3}}+\frac{\bar{\Gamma}_{02}^{\prime}-4 \bar{\beta}_{02}^{e} \bar{\Gamma}_{01}}{16 \varepsilon^{2}}+\frac{\bar{\Gamma}_{02}}{4 \varepsilon}\right)+\mathscr{O}\left(\alpha^{3}\right)
\end{aligned}
$$

Here the bars denote quantities defined in the FDH scheme, $\beta^{e}$ is the $\beta$ function for $\alpha_{e}$, and $\Gamma_{i j}^{\prime}$, $\Gamma_{i j}, \beta_{i j}$ and $\beta_{i j}^{e}$ are the coefficients of $\left(\alpha_{s} / 4 \pi\right)^{i}\left(\alpha_{e} / 4 \pi\right)^{j}$ of the respective quantities.

The FDH formula differs in two respects from the CDR one. First, there are new structures, involving the new $\beta$ function $\beta^{e}$ and involving coefficients of the order $\alpha_{e}$. Second, all quantities are defined in the FDH scheme and thus contain contributions from $\varepsilon$-scalars and differ at the order $N_{\varepsilon}$ from the respective CDR quantities. Ref. [3] has obtained an equivalent formula using a slightly different approach. 

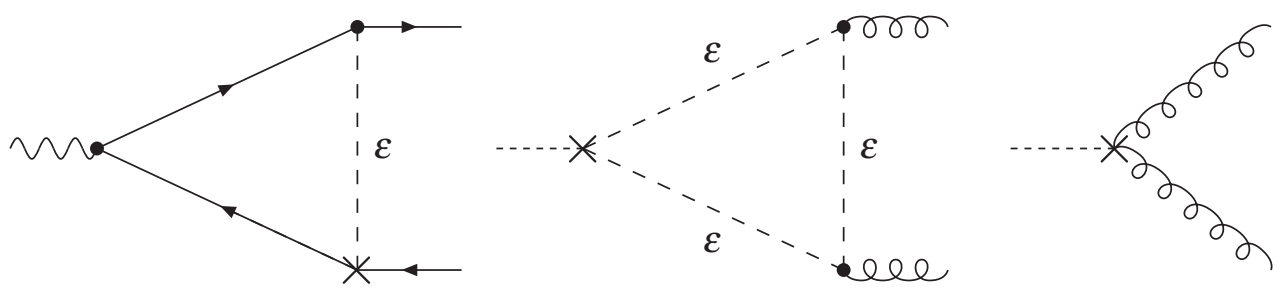

Figure 2: Sample counterterm diagrams contributing to the quark and gluon form factors in the FDH scheme. The crosses denote counterterm insertions involving $\delta \bar{Z}_{\alpha_{e}}^{(1)}, \delta \bar{Z}_{\lambda_{\varepsilon}}^{(1)}$, and $\delta \bar{Z}_{\lambda}^{(2)}$, respectively.

Concrete two-loop computations of form factors serve to test the prediction (3.2) and to determine the unknown coefficients. We have computed the two-loop quark and gluon form factors. In this way $\gamma^{\text {cusp }}, \gamma^{q}$ and $\gamma^{g}$ are determined at the two-loop level, and there are non-trivial checks since the system is overconstrained.

In the actual calculation of the two-loop form factors in the FDH scheme, the correct renormalization is particularly important and non-trivial. We highlight the following two points.

1. Independent couplings of $\varepsilon$-scalars. The couplings of $\varepsilon$-scalars must be treated as independent from the respective couplings of gluons. Fig. 2 shows three sample counterterm diagrams involving the renormalization constants $\delta \bar{Z}_{\alpha_{e}}^{(1)}, \delta \bar{Z}_{\lambda_{\varepsilon}}^{(1)}$, and $\delta \bar{Z}_{\lambda}^{(2)}$, where the upper index denotes the loop order. $\lambda$ is the effective coupling of the Higgs boson to gluons, $\lambda_{\varepsilon}$ is the corresponding coupling to $\varepsilon$-scalars. The renormalization of $\lambda$ also appears in CDR calculations, and here it can be determined in the same way, although the FDH and CDR results differ. The renormalization of $\alpha_{e}$ is known in the literature, but the renormalization of $\lambda_{\varepsilon}$ is new. The $\overline{\mathrm{MS}}$ value of $\delta \bar{Z}_{\lambda_{\varepsilon}}$ can be obtained by an explicit one-loop off-shell calculation of the Higgs $-\varepsilon-\varepsilon$ three-point function.

2. Renormalization schemes $\overline{M S}$ versus $\overline{D R}$. If CDR is used, the $\overline{M S}$ scheme is simply defined by modified minimal subtraction of UV $1 / \varepsilon$ poles. Even in the FDH or DRED regularization schemes, it is possible to choose $\overline{\mathrm{MS}}$ renormalization, implying that renormalized couplings have the same meaning as in CDR-based $\overline{\mathrm{MS}}$. This $\overline{\mathrm{MS}}$ scheme is a natural and advantageous scheme also in the context of FDH and DRED if these regularizations are viewed as advocated here: As long as the $\varepsilon$-scalars are treated as unrelated to gluons but as some new scalar fields which happen to have multiplicity $N_{\varepsilon}$, the $\overline{\mathrm{MS}}$ renormalization scheme simply amounts to minimally subtracting all UV $1 / \varepsilon$ poles, including the ones of the form $N_{\varepsilon} / \varepsilon$ from $\varepsilon$-scalar loops. In this scheme, $\beta$ functions and the $\gamma_{\mathrm{s}}$ involve explicit terms of the $\operatorname{order} N_{\varepsilon}$.

It is also of interest to study the case of $\overline{\mathrm{DR}}$ renormalization. This amounts to setting $N_{\varepsilon}=2 \varepsilon$ and only then minimally subtracting the remaining $1 / \varepsilon$ poles. Renormalized couplings in this scheme differ by finite shifts from the $\overline{\mathrm{MS}}$ couplings.

After consistently renormalizing the form factors either in the $\overline{\mathrm{MS}}$ or $\overline{\mathrm{DR}}$ schemes, we have shown that in both cases the infrared singularities are correctly described by Eq. (3.2). The respective $\beta$ 
and $\gamma$ coefficients differ: in the $\overline{\mathrm{MS}}$ case, these coefficients involve terms of the order $N_{\varepsilon}$; in the $\overline{\mathrm{DR}}$ case, no such terms appear, but the coefficients differ by $\varepsilon$-independent terms. As an illustration, we quote here the results for the quark anomalous dimension. The CDR (plus $\overline{\mathrm{MS}}$ renormalization) results are

$$
\begin{aligned}
\gamma_{10}^{q}= & -3 C_{F}, \\
\gamma_{20}^{q}= & C_{A} C_{F}\left(-\frac{961}{54}-\frac{11}{6} \pi^{2}+26 \zeta(3)\right)+C_{F}^{2}\left(-\frac{3}{2}+2 \pi^{2}-24 \zeta(3)\right) \\
& +C_{F} N_{F}\left(\frac{65}{27}+\frac{\pi^{2}}{3}\right),
\end{aligned}
$$

the results for FDH plus $\overline{\mathrm{MS}}$ renormalization or $\overline{\mathrm{DR}}$ renormalization differ by $\mathscr{O}\left(N_{\mathcal{\varepsilon}}\right)$ or by finite terms, respectively,

$$
\begin{array}{lll}
\bar{\gamma}_{10}^{q}=\gamma_{10}^{q}, & \bar{\gamma}_{10}^{q, \overline{\mathrm{DR}}}=\gamma_{10}^{q}, \\
\bar{\gamma}_{01}^{q}=N_{\varepsilon} \frac{C_{F}}{2}, & \bar{\gamma}_{01}^{q, \overline{\mathrm{DR}}}=0, \\
\bar{\gamma}_{20}^{q}=\gamma_{20}^{q}+N_{\varepsilon}\left(\frac{167}{108}+\frac{\pi^{2}}{12}\right) C_{A} C_{F}, & \bar{\gamma}_{20}^{q, \overline{\mathrm{DR}}}=\gamma_{20}^{q}+\frac{17}{9} C_{A} C_{F}, \\
\bar{\gamma}_{11}^{q}=N_{\varepsilon}\left[\frac{11}{2} C_{A} C_{F}-\left(2+\frac{\pi^{2}}{3}\right) C_{F}^{2}\right], & \bar{\gamma}_{11}^{q, \overline{\mathrm{DR}}}=-\bar{\beta}_{11}^{e, \overline{\mathrm{DR}}} C_{F}, \\
\bar{\gamma}_{02}^{q}=-N_{\varepsilon} \frac{3}{4} C_{F} N_{F}-N_{\varepsilon}^{2} \frac{C_{F}^{2}}{8}, & \bar{\gamma}_{02}^{q, \overline{\mathrm{DR}}}=-\bar{\beta}_{02}^{e, \overline{\mathrm{DR}}} C_{F},
\end{array}
$$

with the non-vanishing $\beta$-coefficients

$$
\begin{aligned}
& \bar{\beta}_{11}^{e, \overline{\mathrm{DR}}}=\left.\bar{\beta}_{11}^{e}\right|_{N_{\varepsilon}=0}=6 C_{F}, \\
& \bar{\beta}_{02}^{e, \overline{\mathrm{DR}}}=\left.\bar{\beta}_{02}^{e}\right|_{N_{\varepsilon}=0}=-4 C_{F}+2 C_{A}-N_{F} .
\end{aligned}
$$

The $\overline{\mathrm{DR}}$ results are relevant since they answer a question of the "Supersymmetry Parameter Analysis" report [12]: the $\overline{\mathrm{DR}}$ scheme is a consistent scheme not only for UV [13] but also for infrared divergences at the multi-loop level.

The $\overline{\mathrm{MS}}$ result has the important application of transition rules between FDH and CDR regularized amplitudes. For the example of the quark form factor $F_{q}$ we can define the combination $Q^{(2)} \equiv F_{q}^{2 l}-\frac{1}{2}\left(F_{q}^{1 l}\right)^{2}$ and obtain

$$
\left[Q^{(2)}-\ln \overline{\mathbf{Z}}_{q}^{(2)}\right]^{\mathrm{FDH}}=\left[Q^{(2)}-\ln \mathbf{Z}_{q}^{(2)}\right]^{\mathrm{CDR}}+\mathscr{O}\left(N_{\varepsilon} \varepsilon^{0}\right),
$$

which allows to translate e.g. an FDH-amplitude into a CDR-amplitude or vice versa.

\section{References}

[1] M. Sperling, D. Stöckinger and A. Voigt, JHEP 1307 (2013) 132; JHEP 1401 (2014) 068.

[2] C. Gnendiger, A. Signer and D. Stöckinger, Phys. Lett. B 733 (2014) 296. 
[3] W. B. Kilgore, Phys. Rev. D 86 (2012) 014019.

[4] A. Signer and D. Stöckinger, Phys. Lett. B 626 (2005) 127; Nucl. Phys. B 808 (2009) 88.

[5] P. H. Chankowski, S. Pokorski and J. Rosiek, Nucl. Phys. B 423 (1994) 437; A. Dabelstein, Z. Phys. C 67 (1995) 495.

[6] E. Kraus and K. Sibold, Z. Phys. C 68 (1995) 331; E. Kraus, Annals Phys. 262 (1998) 155; W. Hollik, E. Kraus, M. Roth, C. Rupp, K. Sibold and D. Stöckinger, Nucl. Phys. B 639 (2002) 3.

[7] F. Staub, Comput. Phys. Commun. 182 (2011) 808; Comput. Phys. Commun. 184 (2013) 1792; Comput. Phys. Commun. 185 (2014) 1773.

[8] P. Athron, J. -h. Park, D. Stöckinger and A. Voigt, arXiv:1406.2319 [hep-ph].

[9] T. Becher and M. Neubert, Phys. Rev. Lett. 102 (2009) 162001 [Erratum-ibid. 111 (2013) 19, 199905]; JHEP 0906 (2009) 081 [Erratum-ibid. 1311 (2013) 024];

[10] E. Gardi and L. Magnea, JHEP 0903 (2009) 079; Nuovo Cim. C 32N5-6 (2009) 137.

[11] W. B. Kilgore, Phys. Rev. D 83 (2011) 114005.

[12] J. A. Aguilar-Saavedra et al., Eur. Phys. J. C 46 (2006) 43 [arXiv:hep-ph/0511344].

[13] D. Stöckinger, JHEP 0503 (2005) 076 [arXiv:hep-ph/0503129]. 